\title{
Prospección en el sitio arqueológico de Pacopampa*
}

Julián I. Santillana

Por encargo del Seminario de Historia Rural Andina de la Universidad Mayor de San Marcos, se realizó un reconocimiento arqueológico en Pacopampa y en los alrededores del distrito de Querocoto, provincia de Chota, durante los meses de julio y agosto del presente año (1975).

Sin embargo, fuimos un poco más allá. En la esperanza de encontrar nuevos derroteros para la investigación en Pacopampa, realizamos una prospección etnológica y etnohistórica en pueblos que antaño sirvieron de asentamiento a etnias hoy desaparecidas. Además, revisamos algunos archivos en las alcaldías y notarías de Querocoto, Huambos y Chota.

Sea este informe "en bruto" la muestra de reconocimiento al Dr. Pablo Macera, con quien poco me he comunicado y nada nos conocemos, pero en quien intuyo una profunda preocupación -casi desesperadaporque no se pierda la historia social de los pueblos andinos.

* Publicado originalmente por el Seminario de Historia Rural Andina, Lima, 1975. 


\section{Estrategia de investigación}

La arqueología por si sola dice muy poco. Centrarse en la estrechez técnica conduce sólo a un absurdo tecnicismo asocial. Quien escribe este informe cree fervientemente en la investigación interdisciplinaria. Sólo así se encuentran nuevos derroteros y alternativas para enfrentar un problema concreto. Esto es válido en todas las ciencias sociales. En algunos casos la investigación arqueológica parte de un indicativo hallado en un manuscrito etnohistórico (Visita de Huámachuco... Proyecto Huanuco Viejo, 1966) o de una referencia personal etnológica; y a partir de esto se busca el sitio con respecto a la interrogante arqueológica. En el caso específico de Pacopampa se parte de la evidencia monumental, "El Templo de Pacopampa". Se empezó ahí y ahí se quedó. A falta de mayor conocimiento del sitio se tuvo la impresión parcial y unilateral de que sólo es un templo fabuloso.

Para esta investigación y otras se debe plantear una estrategia de investigación. Afortunadamente se tuvo la oportunidad de reconocer la zona y se corroboró la gran importancia del templo y de otros sitios palaciegos. Se planteó entonces una estrategia de investigación; y para encarar los problemas se estructuró tácticas a seguir.

A continuación reproducimos el esquema (véase el cuadro 1).

Esta estrategia de investigación conlleva una identidad ideológica del investigador, quien trazará los planes y enfrentará el problema, es decir, una metodología que implica raciocinio teórico sobre problemas generales. Las técnicas sirven sólo como atributo dentro de esa metodología.

\section{Ecología y asentamiento}

Los yacimientos arqueológicos ubicados en Pacopampa están comprendidos dentro de una base geográfica formada por el río Chotano por el este; Paltic-Inguerayacu por el norte y noroeste, y el Huamboyacu-Obraje por el Sur y Sureste; forma típica de archipiélagos norandino.
Esta área está formada por una macizo montañoso en forma semicircular (casi de herraje) que va de Sur a Norte, de cuyo centro se desprende un eje que forman las montañas de Pacopampa. Ramal en el que se halla el actual pueblo de San Pedro y el "Templo de Pacopampa", cuya ubicación es por todos lados estratégica y privilegiada. El terreno varía desde los 1000 hasta montañas de $2900 \mathrm{msnm}$.

El macizo montañoso presenta dos características fundamentales: 1) por el lado oeste, presenta una caída perpendicular hasta dar con el río Paltic en el que no existen faldas anchas o terrenos con posibilidades de cultivo ni de asentamientos en grande; 2) por el lado este, la naturaleza es más pródiga. Presenta un ecosistema casi generalizado, apto para un asentamiento prolongado y generacional. Posee zonas de cultivo y riego propio. Las zonas de cultivo están formadas por la cuenca de los ramales que se desprenden del macizo montañoso y el eje central de Pacopampa.

Visto como un todo, encierra tres pisos ecológicos definidos. Para ello conservamos la misma diferencia y definición que hace el indígena de la zona: Playa, Quechua y Jalka. Estas categorías se diferencian en algo del nombre de la racionalización climática elaborada por Pulgar Vidal.

$\begin{array}{ll}\text { En Pacopampa } & \text { En Pulgar Vidal } \\ \text { La Playa } & \text { La Yunga } \\ \text { Quechua } & \text { Quechua } \\ \text { Jalka } & \text { Suni }\end{array}$

\subsection{Playa}

De 1000 a 1200 msnm. Fajas estrechas a orillas del río Chotano. Intenso calor, nada de viento. Bajo porcentaje de pluviosidad anual. Cultivos sólo con riego. Variedad de árboles frutales y de pan llevar: caña de azúcar, yuca, frijoles, pastos, guarangos, plátanos, chirimoyas, café, algodón, arroz, papayas. Animales: aves de corral, caballos, puercos, vacas. Población móvil y escasa, generada por la aparición y desapari- 


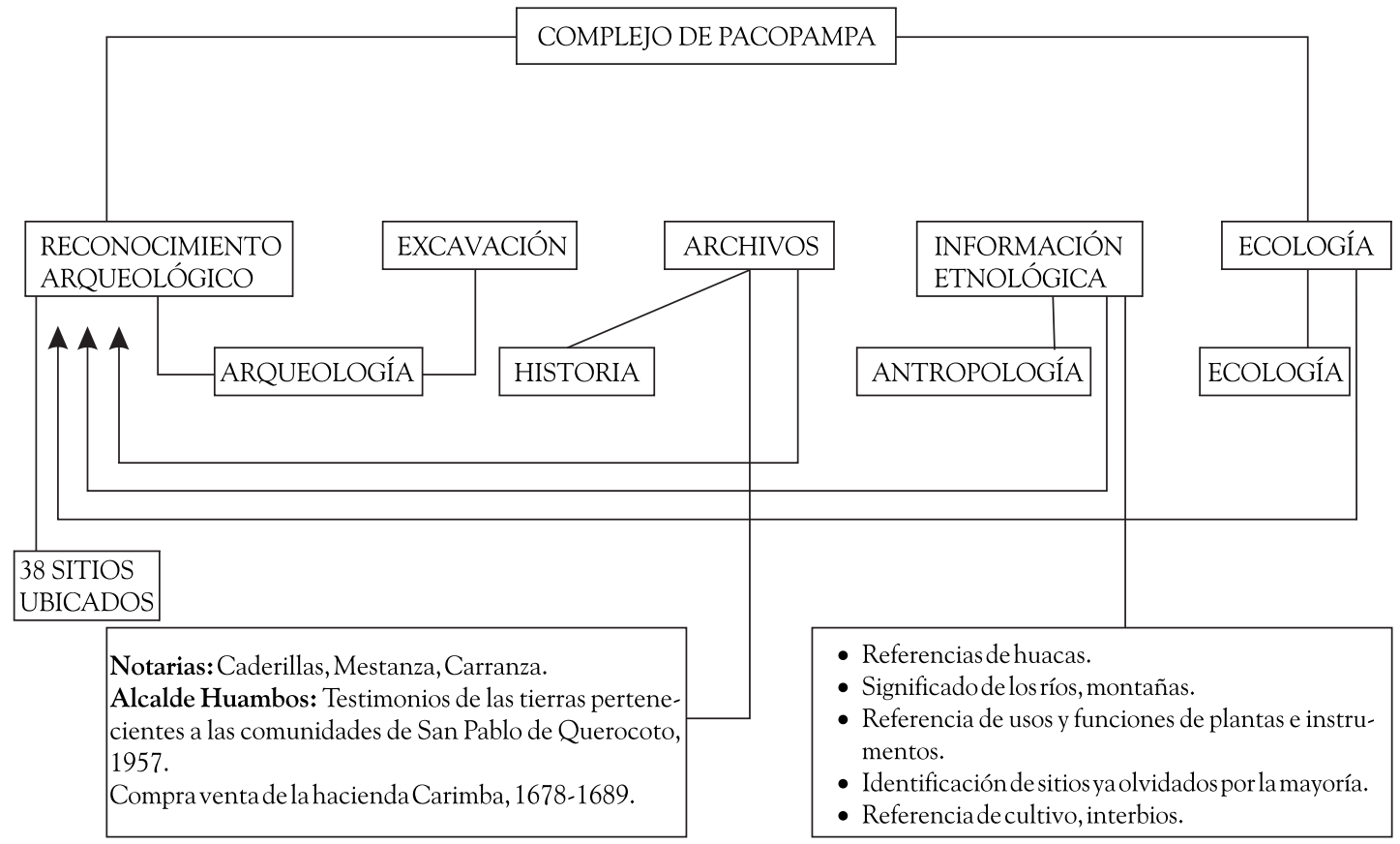

Cuadro 1. Diagrama de estrategia de investigación.

ción repentina de tierras aluviales a consecuencia de las crecidas de aguas del río.

Como podemos apreciar, no hay casi una planta que no haya sido traída de afuera. Y si procedemos a una retrospección histórica, a sociedades de no-mercado, la encontraríamos no apta para un poblamiento permanente e incapaz de abastecer a una población relativamente grande, donde existe cierto equilibrio etno-ecológico, como sí existe en la zona Quechua y que veremos más adelante.

Tal es así, que fue casi imposible un asentamiento formativo en dicho piso ecológico de la zona. Pero sí hay asentamientos posteriores por sociedades tanto complejas como por estados regionales: la llamada cultura Cajamarca, de la cual sí se halló evidencia (Pa-029. Hasta ahora sólo un yacimiento: "La naranjita de Guangapucara”).

Pero lo dicho en líneas arriba, no quita la posibilidad de un dominio de este piso ecológico para abastecer, por ejemplo, de algodón por parte de pobladores de la Quechua y Jalka en épocas pri- mitivas y/o posteriores también; y que quizás poblaron estacionariamente con estos fines. La continuación del reconocimiento arqueológico nos podrá aclarar definitivamente este problema.

Dentro de este patrón de poblamiento ribereño también existe un sitio muy pequeño ( $\mathrm{Pa}$ 030), que no presenta cerámica superficial. Debe haber, pero lo enmarañado de la vegetación y la grama impidieron hallarla.

Otro de los sitios ubicados (Pa-028. "El Gigante") es muy importante porque abarca dos pisos ecológicos. Es un sistema de graderías o terrazas que empiezan en el río Chotano y trepa hasta la Quechua, termina en una planicie cultivada y regada por dos canales de agua que nacen en "los ojos de agua", ubicados en la partes altas de las graderías. Cada gradería está preparada por unos $30 \mathrm{~m}$ más o menos. La producción actual es de maíz.

Sea cual fuere el pueblo que la construyó y utilizó, queda claro el doble dominio ecológico muy visible a todos los ojos. 


\subsection{Quechua}

De los 1200 a 2200 msnm. Provista de un clima templado. Agricultura de riego. Extensiones considerables para el cultivo. Con mayores posibilidades de agricultura extensiva. Los árboles frutales y de pan llevar producen bien cuando tienen riego. Igualmente el ganado estará en buenas condiciones si es que tiene pasto, el cual se produce gracias al riego. En la actualidad han abierto nuevos canales de agua que vienen desde la Jalka y riegan las partes altas y centrales de este piso ecológico. Las partes más bajas, hasta los límites de la Playa no tienen riego, pero son potencialmente productivas. A medida que el indígena es desplazado por la miniparcelación, va abriendo nuevas chacras en estas zonas, hoy sin riego. Pero en cuanto construyen una acequia la tierra se torna productiva. Ahora bien, en todas estas zonas -hoy deshabitadas y en proceso de habitación- hay evidencias de población antigua. Así lo determina el reconocimiento arqueológico (28 sitios) entre núcleos mínimos (Pa-07 01, 03, 04, etc.), núcleos medios ( $\mathrm{Pa}$ 05, 05, etc.) con construcciones y sitios ceremoniales o de algún fin religioso ( $\mathrm{Pa}-14,06$ 16), que han servido de asentamiento a una población relativamente densa que tenía que cultivar y trabajar. ¿Cómo pudieron producir éstas tierras secas? La única posibilidad es el riego ¿Y el agua? Estaba en "los ojos de agua" y lagunas (naturales y artificiales) de la Jalka.

En este archipiélago de Pacopampa encontramos seis micro-asientos con todas las posibilidades de producción económica agrícola. Cuatro perfectamente definidos y tres de ellos bien reconocidos (Fig. 3).

Los micro-asientos parten del eje central de Pacopampa (Figs. 2 y 3) y se orientan al Este, extendiéndose hasta las partes altas del río Chotano. Estas cuencas son aptas para el cultivo. En ellas se hallaron muy pocos yacimientos arqueológicos más no así en las partes altas de los ramales que forman estos micro-asientos (ver mapa general de ubicación de sitios). Es decir, supieron aprovechar al máximo las cuencas o partes bajas para el cultivo, y por seguridad para viviendas, las lomas.

\subsection{Jalka}

De los 2200 a más. Ecosistema especializado. Las posibilidades agrícolas son muy bajas. Sólo produce pasto, arracacha y en los últimos años papa. La helada es frecuente. Mayores posibilidades para el pastoreo. Los hombres dependen aquí del intercambio de productos pecuarios por productos agrícolas de la Qechua y la Playa, pero principalmente se abastecen en los mercados de Querocoto y Pacopampa. La densidad poblacional aumenta cada día. En esta zona existe un núcleo mayor de asentamiento que abarca casi 8 kilómetros de largo en las lomas de los cerros que componen el macizo montañoso. Estos sitios arqueológicos presentan una arquitectura palaciega: piedras talladas y pulidas y más de 200 evidencias desde la loma de "Los Gentiles", pasando por la "La Alfombrilla", la Totora, Vista Alegre, Cachen, el Rollo y el Palmal (ver mapa general). Además, estos centros están próximos a las lagunas, "ojos de agua" y canales que hay en la Jalka.

En las faldas del cerro Totora se observan tres plataformas grandes de unos $150 \mathrm{~m}$ cada uno ¿Sirvieron para la agricultura?.

Toda esta zona pertenece solo a un complejo. La loma donde está asentada esta población posee gran visibilidad a las quebradas de Pacopampa, Querocoto, Cerro Negro, y el asiento que queda al Suroeste del Totora. De allí provienen las cabezas clavas y otras representaciones en piedras que se hallan en Querocoto y Chiclayo. El tipo de construcción es de piedras grandes a los costados y relleno al centro.

Sin alejarnos de nuestro cuestionamiento fundamental, planteamos algunas preguntas complementarias. Hoy, que las relaciones de dominio de pisos ecológicos han encontrado un intermediario llamado mercado (mercancíadinero) aunque sea en una reproducción simple, 
han variado las relaciones sociales, tornándolas en paralelas, lo que ya no encierra un contexto vertical del usufructo. Lo que no ocurría antes, ni tampoco creemos en esa época formativa, donde los pobladores que habitaban viviendas suntuosas, en tierras que ecológicamente no eran aptas para el cultivo o por lo menos un cultivo variado, sí tenían el dominio de las fuentes del agua, ¿cómo podían sostenerse sólo con un producto? Por cierto que no, entonces tenían que abastecerse de los productos de las zonas bajas, económicamente productivas en las que probablemente estaban asentadas poblaciones que ocupaban viviendas sencillas.

A esta altura aclararemos lo siguiente: que el raciocinio llevado hasta aquí se confirma porque la cerámica hallada en todos estos sitios de los tres pisos ecológicos se parecen mucho, pero ia qué fase pertenecen? Todavía no sabemos. Lo que si está claro es que pertenecen a una misma época.

Una observación más. Este énclave de Pacopampa no se abastecía en su totalidad y exclusivamente de los pisos ecológicos descritos, sino que rompió y buscó nuevas fronteras productivas para satisfacer necesidades básicas ornamentales. El oro por ejemplo. El yacimiento más cercano, en el que actualmente se está iniciando la explotación, se halla en dos montañas antes de la desembocadura del Chotano en el río Chamaya, en la margen izquierda, y creemos que sea ese el yacimiento abastecedor, porque cerca de esas minas hay "ruinas con piedras iguales a San Pedro, subiendo el río Calucán”. 'Sugeriremos una explotación de la zona.

Para aclarar más el privilegio ecológico del "enclave Pacopampa", cabe recordar que Querocotillo, distrito colindante con Pacopampa, vive a expensas de éste último. Querocotillo no tiene riego. El poco maíz que produce sólo es en época de lluvias. La arracacha y papa las llevan de Pacopampa. Igualmente los cerros que suben Mollebamba, circundan Huambos, descienden a Cochabamba y Lajas. Y por el otro lado hasta Yanacuna son áridos, cálidos y rocosos.

El medio ambiente en las faldas del Ashahuillca y en los bajos del cerro Negro, pasando por las faldas de Pacopampa, nos ayuda a explicar el por qué no se encontró más sitios arqueológicos en estas zonas casi imposibles para la agricultura.

Este límite ecológico entre cada piso se percibe por la vegetación que existe. Aunque con el transcurso del tiempo se va alterando, por ejemplo, en Pacopampa se van adaptando (idomesticando?) plantas de otros ambientes, como la cabuya de las quebradas profundas.

\section{Supuestos arqueológicos}

El complejo de Pacopampa se inscribe como un centro de poder dentro de una red de enclaves asentados en ecologías parecidas, con aproximación en sus contactos y tiempo de desarrollo, sin perjuicio de los posibles desarrollos autónomos de la costa y sierra. Y así como Pacopampa, lo es Chavín y otros centros que deben buscarse en el nor-ande peruano y más allá de esos límites.

Centro de poder basado en un despotismo agrario-teocrático en el que un sector de la población ejercía un control del sistema de aguas; desde su nacimiento en los "ojos de agua”, pasando por la acumulación en pozos y/o lagunas naturales y/o artificiales hasta su distribución. Despotismo agrario-teocrático generado por la posibilidad de una función dual: a) como centro ceremonial; b) como núcleo central de distribución de aguas prestada por el gran centro artificial conocido como "El Templo de Pacopampa"

Casi siempre el agua constituye la ligación base en las relaciones económicas de la sociedad andina y probablemente de otras.

\footnotetext{
1 Información personal de tipo copia.

2 Pudimos evidenciar casi todo un sistema de lagunas y canales por toda el área reconocida. Así tenemos: restos
} 
Evidentemente que lo agrario no priva la premisa primaria de lo religioso en la gestación de estos poderes (Schaedel 1956; Adams, 1966).

El sector que ejercía control sobre las aguas puede haberse asentado en las zonas donde existe una arquitectura palaciega, como La Alfombrilla, La Totora, desde donde cumplían las funciones de poder. Si ésta imagen es correcta, habría que buscar la residencia sacerdotal y divina ¿junto a los templos o fuera de ellas? o ¿qué?

Otra de las cuestiones a resolver se refiere a la influencia que ejerció la ceja de selva en esta época. En principio, aunque se admite esa gran influencia ecológica ejercida durante esta época formativa, hay que ver la particularidad de algunos de sus componentes. Un análisis ecológico del arte es aquí pertinente. La serpiente y el felino son seres representados en diversas formas naturales estilizadas y en diversos objetos. Creemos que la función desempeñada fue tal vez decisiva, más que importante, en tanto seres que constituyen los miembros bio-tipos en la selva, reflejados posteriormente como miembros supraestructurales ideo-tipo.

$\begin{array}{lll}\text { Ideológico } & \text { ideo-tipo } & \text { felino } \\ \text { Biológico } & \text { bio-tipo } & \text { serpiente }\end{array}$

Idealizados y llevados a categorías abstractas, y por qué no, con fines de temor y poder.
Materializados y plasmados en sus más representativas obras de arquitectura palaciega religiosa y otros objetos de importancia.

Concluyendo, el monumental templo de Pacopampa se debe ver como un centro religioso e hidráulico, alimentado por los núcleos mínimos y medios, y relacionado con lazos mínimos y medios directos y máximos indirectos.

\section{Proyección social}

Durante la permanencia de Pacopampa se desarrolló en lo posible la relación universidadpueblo, teniendo en cuenta la tarea de transmitir el conocimiento adquirido y prestando ayuda en la medida de las posibilidades. De este modo se hizo lo siguiente:

1) Compra y donación de un terreno a la Universidad de San Marcos para que se construya: a) un museo sitio; b) una posada para los investigadores; (adjuntamos el bosquejo del plano); d) Centro Cívico Comunal.

2) Elaboración de plano de la Plaza de Armas de Pacopampa. Éste era un gran deseo de los pobladores pertinentes para conseguir ayuda de las instituciones pacopampinas de Lima y otras ciudades.

3) Planificación y construcción del monumento al felino, constituyendo este una forma más de recuperar el valor de las manifestaciones culturales andinas. Para esto hemos procurado representar -en lo posible simbólicamente- el

de canales en el actual trayecto de la toma de agua de las Rimana y Peña Blanca. Este canal tiene $8.70 \mathrm{~km}$ de largo hasta llegar a Pacopampa. Tres lagunas, casi secas, de más o menos $30 \mathrm{~m}$ de diámetro ubicadas en la loma de Vista legre y la falda lateral (Norte) del cerro Totora. Un canal por la loma entre Vista Alegre, Cachén y La Playa a 2 km del templo. En la actualidad han abierto un nuevo canal para abastecer de agua a Querocoto en el cual "hemos roto canales antiguos, por el cerro Negro y el Tendal" (Informe personal de U. Vásquez). Por la loma del eje central de Pacopampa existe un resto de canal, que tal vez recibía las aguas de los canales de las partes altas. En Cochecorral "se ha encontrado dos canales, uno grande y otro pequeño con grava en la base" (información personal de A. Peralta). Igualmente en los terrenos de la señora Isolina Mendoza hay canales que atraviezan una parte de la carretera. Del mismo modo M. Farro excavó y "halló un canal que conducía a un hueco profundo..." (Información personal de A. Peralta). Entre el Mirador y la primera plataforma existe una laguna que G. Alarcón dice "... que antes salía una canal que usaban para regar la parte baja... pero como se cayeron algunos ganados, la rellenaron con piedras...”. Además de los canales-galerías del templo orientados hacia los micro-asientos descritos, en la loma de "los Alisos" se encontraron restos de canales que bajaban a Carayuc. (Información personal de G. Alarcón). 
cerro y la plataformas del Templo de Pacopampa, empleando materiales de la zona.

4) Plano y ejecución de un letrero de $3 \times 2$ metros con una leyenda sobre las ruinas y el plano de la misma.

5) A pedido de las autoridades del distrito de Querocoto se levantó el plano topográfico de los terrenos del Colegio Nacional de Querocoto, en los cuales se construirá el local del mencionado colegio.

6) Confección de programa festivo para el aniversario de la fiesta patronal de Pacopampa. Adjuntamos una copia.

7) Preparación de dos obras teatrales de temática cultural, que se presentó en las fiestas jubilares de Pacopampa.

8) Iniciativa y preparación de la primera Feria Agropecuaria en Pacopampa ya que no se realizará ninguna en los distritos de la zona. Para ello se donó seis diplomas para los participantes y se proyectó para el próximo año el premio "La Arracacha de Pacopampa”, que será el máximo premio de la Feria. Éste consiste en un trofeo de $20 \mathrm{~cm}$ de tamaño, donde esté representado una planta de la arracacha, producto muy periférico y abundante en Pacopampa.

9) Las piedras como "el Rastro", "las columnas", "el rollo", "las serpientes" fueron alzadas sobre otras piedras pequeñas y techadas luego, para protegerlas del sol, la humedad y la erosión posterior.

10) Conferencia dictada a los alumnos y docentes secundarios del distrito de Querocoto sobre las investigaciones que se realizan.

11) Conferencia dictada en el programa de sociología de la Universidad de Lambayeque sobre El desarrollo de la sociedad andina y sus implicancias en la formación de la conciencia nacional.

12) Participación en todas las actividades y orientación de los clubes culturales de Pacopampa.

Fueron estas las actividades que se desarrollaron durante los meses de julio y agosto.

\section{A manera de sugerencia}

Después de permanecer en Pacopampa se sugiere lo siguiente:

1) Sacar dos órdenes conminatorias de la autoridad respectiva para los señores Francisco Palacios (profesor primario) y Máximo Farro, las que deben ser entregadas por intermedio de la Guardia Civil de Querocoto, a fin de que no continúen incrementando sus colecciones.

2) Designar tareas concretas, cada cierto tiempo, al guardián de las ruinas para avanzar en la seriación del sitio.

3) Construir un nuevo canal de agua que no atraviese las ruinas, porque en la actualidad está erosionando considerablemente las estructuras arqueológicas que existen en ésta. Para esto se cree conveniente que se lo construya por medio de una "minka", y hacer sentir a la gente que es una cosa de ellos, que les pertenece, y no como alguna vez se pensó que el alcalde debía mandar a que trabajen.

4) Hacer algo para que el material depositado por lo señores Rosas en casa de Críspulo Tapia, sea sacado de ahí, porque se nos comunicó "... que ya era mucho tiempo y que necesitaban esas habitaciones...".

5) Continuar con la explotación arqueológica siguiendo la cuenca del Chotano (aguas arriba y abajo) y si fuera posible ampliar más hacia el Noreste, buscando y siguiendo luego la cuenca de otro río ya en la selva.

6) Incluir dentro del plan de excavaciones los sitios de "La Alfombrilla", Totora, Cachén, el Naranjo, Guayachucho, saliendo del tradicional sitio monumental o por lo menos paralelamente.

7) Ampliar el campo de estudio de la zona e integrar al trabajo arqueológico un etnohistórico y etnológico.

8) Finalmente (léase primeramente) se estructura un Cuadro General de Trabajos del Proyecto Pacopampa, porque en la actualidad existe cierto aislamiento entre los arqueólogos que participan en los trabajos. 


\section{Cuadro 1}

Registro de exploración: Querocoto, Querocotillo, Humabos, provincia de Chota

\begin{tabular}{|c|c|c|c|}
\hline Sitio & Nombre & Anexo & Altitud \\
\hline Pa-001 & Loma del Membrillo & Succhurán & $2,090 \mathrm{~m}$ \\
\hline $\mathrm{Pa}-002$ & $i$ & Succhurán & $2,010 \mathrm{~m}$ \\
\hline $\mathrm{Pa}-003$ & Yuayampampa & Sigues & $2,050 \mathrm{~m}$ \\
\hline $\mathrm{Pa}-004$ & LaQueda & Sigues & $1,765 \mathrm{~m}$ \\
\hline $\mathrm{Pa}-005$ & ElNaranjo & Sigues & $1,490 \mathrm{~m}$ \\
\hline $\mathrm{Pa}-006$ & ElMirador de Molompaque & Sigues & $1,550 \mathrm{~m}$ \\
\hline $\mathrm{Pa}-007$ & LaConga & LaRaya & 1,950 \\
\hline $\mathrm{Pa}-008$ & $?$ & LaRaya & 2,150 \\
\hline $\mathrm{Pa}-009$ & Machaypungu & Sigues & 1,850 \\
\hline $\mathrm{Pa}-010$ & Loma de los Alisos & Carayuc & 2,340 \\
\hline $\mathrm{Pa}-011$ & $?$ & AguaBlanca & 2,320 \\
\hline $\mathrm{Pa}-012$ & ElRejo & Sahuindo & 2,200 \\
\hline $\mathrm{Pa}-013$ & Chilalan & Sahuindo & 2,050 \\
\hline $\mathrm{Pa}-014$ & LaFila & Limón & 1,950 \\
\hline $\mathrm{Pa}-015$ & LaGuachuma & Limón & 1,826 \\
\hline $\mathrm{Pa}-016$ & Cundín & Paraguay & 2,300 \\
\hline $\mathrm{Pa}-017$ & Cachón & Vista Alegre & 2,530 \\
\hline $\mathrm{Pa}-018$ & Vista Alegre & Vista Alegre & 2,520 \\
\hline $\mathrm{Pa}-019$ & La Totora & Querocoto & 2,690 \\
\hline $\mathrm{Pa}-020$ & Totora Alta & Querocoto & 2,800 \\
\hline $\mathrm{Pa}-021$ & LomadelCachín & Vista Alegre & 2,553 \\
\hline $\mathrm{Pa}-022$ & ElRollo & Vista Alegre & 2,570 \\
\hline $\mathrm{Pa}-023$ & LaPalma & AguaBlanca & 2,570 \\
\hline $\mathrm{Pa}-024$ & LaPunta de la Palma & AguaBlanca & 2,520 \\
\hline $\mathrm{Pa}-025$ & SanPablo & San Pablo & 2,520 \\
\hline (x) $\mathrm{Pa}-040$ & Pandanche & Sigues & $?$ \\
\hline (x) $\mathrm{Pa}-041$ & Ruinas Pacopampa & Pacopampa & $?$ \\
\hline $\mathrm{Pa}-026$ & Loma de Ñaupa Llacta & San Pablo & 2,485 \\
\hline $\mathrm{Pa}-027$ & Los Canches & San Pablo & 2,320 \\
\hline $\mathrm{Pa}-028$ & ElGigante & Sinchimache & 1,120 \\
\hline $\mathrm{Pa}-029$ & La Naranjita de Guanca Pucará & Sinchimache & 3,180 \\
\hline $\mathrm{Pa}-030$ & $?$ & Limón & $1,092 \mathrm{~m}$ \\
\hline
\end{tabular}


Pa-03

$\mathrm{Pa}-032$

$\mathrm{Pa}-033$

Pa-034

$\mathrm{Pa}-035$

Pa-036

$\mathrm{Pa}-037$

Pa-038

Pa-039

Pa-040

$\mathrm{Pa}-041$

$\mathrm{Pa}-042$

$\mathrm{Pa}-043$

$\mathrm{Pa}-044$

$\mathrm{Pa}-045$

$\mathrm{Pa}-046$

$\mathrm{Pa}-047$

$\mathrm{Pa}-048$ ()

$\mathrm{Pa}-049$ ()

$\mathrm{Pa}-050$ (ㅇ)

$\mathrm{Pa}-051$ ()

$\mathrm{Pa}-052(\stackrel{\circ}{)}$

$\mathrm{Pa}-053$ () $^{\circ}$

$\mathrm{Pa}-054\left(^{(}\right)$

$\mathrm{Pa}-055$ ()

$\mathrm{Pa}-056\left(^{\circ}\right)$

$\mathrm{Pa}-057$ ()

$\mathrm{Pa}-058$ ()

$\mathrm{Pa}-059$ (9)

$\mathrm{Pa}-060$ (9)

$\mathrm{Pa}-061(\stackrel{\circ}{)}$

$\mathrm{Pa}-062$ ()

$\mathrm{Pa}-063$ ()

$\mathrm{Pa}-064\left(^{\circ}\right)$

$\mathrm{Pa}-065$ ()

$\mathrm{Pa}-066\left(^{(9}\right)$

$\mathrm{Pa}-067(\stackrel{\circ}{)}$
Loma de Guayampampa

Loma de los Vientos

Guayachucchu

LosLoritos

La LomaGrande

Yerbabuena

La Fila de la Yerbabuena

Loma delPayacul

Cerro Negro

Hda. Pariamarca

AlAtajo

Marabamaba

LaLomade Mitobamba

Lima Pampa

Hda. Sillangata

Paria

Co-Huambo

Champicirca

Incahuasi

ElPalmal

Incahuasi

Cachipampa

Hda. Quipacuc

LaPaccha

Ninabamba

Las Torrecitas

Cueva del Co Amachay

Cueva del $\mathrm{C}^{\mathrm{o}}$ La Perita

Chicopón

Isco

Negropampa

Chocmarca

Churucancha

Condormarca

Rejopampa

Co-Coyunde

Huambos Viejo
Guayampampa

$2,210 \mathrm{~m}$

Querocoto

$2,180 \mathrm{~m}$

Querocoto

$2,280 \mathrm{~m}$

Ayanchacra

$1,640 \mathrm{~m}$

Ayanchacra

$2,020 \mathrm{~m}$

Agua Blanca

$2,000 \mathrm{~m}$

AguaBlanca

$1,940 \mathrm{~m}$

Payacul

$2,150 \mathrm{~m}$

Querocoto

$3,204 \mathrm{~m}$

Pariamarca

$2,500 \mathrm{~m}$

Campam. Rocoto

$2,495 \mathrm{~m}$

Maraybamba

Mitobamba

Querocotillo

Sillamgata

Paria

Paltic

Huambos

Huambos

Santa Rosa

Mollebamba

CoPiedras Qanes

Sn. Luis

Yatumpampa

Ninabamba

Chiguivip

Llama

Llama

Llama

Llama

Chota

Chota

Chota

Chota

Chota

Chota

Huambos

?

$?$

$2,020 \mathrm{~m}$

?

?

$2,850 \mathrm{~m}$

?

?

$2,500 \mathrm{~m}$

?

?

?

?

?

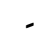




\section{Cuadro 2}

Ubicación de los pisos ecológicos y características fundamentales

\begin{tabular}{|c|c|c|c|c|c|c|c|}
\hline Sitios & Jalka & Quechua & Playa & $\begin{array}{l}\text { Estruc. } \\
\text { Esterx. }\end{array}$ & Cerámica & Lítico & $\begin{array}{l}\text { Aprox. } \\
\text { Esterx. }\end{array}$ \\
\hline $\mathrm{Pa}-001$ & - & $\mathrm{X}$ & - & - & $\mathrm{X}$ & - & 2002 \\
\hline $\mathrm{Pa}-002$ & - & $\mathrm{X}$ & . & - & $\mathrm{X}$ & - & 1002 \\
\hline $\mathrm{Pa}-003$ & - & $\mathrm{X}$ & - & - & $\mathrm{X}$ & - & 4002 \\
\hline $\mathrm{Pa}-004$ & - & $\mathrm{X}$ & - & - & $\mathrm{X}$ & - & 2002 \\
\hline $\mathrm{Pa}-005$ & - & $\mathrm{X}$ & - & $\mathrm{X}$ & $\mathrm{X}$ & - & $200 / 100$ \\
\hline $\mathrm{Pa}-006$ & - & $\mathrm{X}$ & - & - & $\mathrm{X}$ & - & 5002 \\
\hline $\mathrm{Pa}-007$ & - & $\mathrm{X}$ & - & $\mathrm{X}$ & $\mathrm{X}$ & - & $100 / 60$ \\
\hline $\mathrm{Pa}-008$ & - & $\mathrm{X}$ & - & $\mathrm{X}$ & $\mathrm{X}$ & - & $60 / 30$ \\
\hline Pa-009 & - & $\mathrm{X}$ & - & $\mathrm{X}$ & $\mathrm{X}$ & - & $80 / 80$ \\
\hline $\mathrm{Pa}-010$ & $\mathrm{X}$ & - & - & $\mathrm{X}$ & $\mathrm{X}$ & - & $50 / 30$ \\
\hline $\mathrm{Pa}-011$ & $\mathrm{X}$ & - & - & $\mathrm{X}$ & $\mathrm{X}$ & - & $100 / 50$ \\
\hline $\mathrm{Pa}-012$ & - & $\mathrm{X}$ & - & - & $\mathrm{X}$ & - & $30 / 30$ \\
\hline $\mathrm{Pa}-013$ & - & $\mathrm{X}$ & - & - & $\mathrm{X}$ & - & $300 / 30$ \\
\hline $\mathrm{Pa}-014$ & - & $\mathrm{X}$ & - & $\mathrm{X}$ & $\mathrm{X}$ & - & $80 / 60$ \\
\hline $\mathrm{Pa}-015$ & - & $\mathrm{X}$ & - & - & $\mathrm{X}$ & - & $100 / 100$ \\
\hline $\mathrm{Pa}-016$ & $\mathrm{X}$ & - & - & $\mathrm{X}$ & $\mathrm{X}$ & - & $30 / 3$ \\
\hline $\mathrm{Pa}-017$ & $\mathrm{X}$ & - & - & $\mathrm{X}$ & $\mathrm{X}$ & - & $80 / 30$ \\
\hline $\mathrm{Pa}-018$ & $\mathrm{X}$ & - & - & $\mathrm{X}$ & $\mathrm{X}$ & - & $100 / 40$ \\
\hline $\mathrm{Pa}-019(\mathrm{X})$ & $X$ & - & - & $\mathrm{X}$ & $\mathrm{X}$ & - & $200 / 100$ \\
\hline $\mathrm{Pa}-020$ & $\mathrm{X}$ & - & - & - & $X$ & - & $80 / 2$ \\
\hline $\mathrm{Pa}-021$ & $\mathrm{X}$ & - & - & $\mathrm{X}$ & $\mathrm{X}$ & - & $60 / 60$ \\
\hline $\mathrm{Pa}-022$ & $\mathrm{X}$ & - & - & $X$ & $X$ & - & $60 / 60$ \\
\hline $\mathrm{Pa}-023$ & $\mathrm{X}$ & - & - & $\mathrm{X}$ & $X$ & $\mathrm{X}$ & $50 / 40$ \\
\hline $\mathrm{Pa}-024$ & $\mathrm{X}$ & - & - & $X$ & $X$ & - & $100 / 20$ \\
\hline $\mathrm{Pa}-025$ & $\mathrm{X}$ & - & - & $X$ & $\mathrm{X}$ & - & $60 / 30$ \\
\hline $\mathrm{Pa}-026$ & $\mathrm{X}$ & - & - & $\mathrm{X}$ & $\mathrm{X}$ & - & $80 / 60$ \\
\hline $\mathrm{Pa}-027$ & $\mathrm{X}$ & - & - & $\mathrm{X}$ & $X$ & - & $100 / 80$ \\
\hline $\mathrm{Pa}-028$ & - & - & $X$ & $X$ & $X$ & - & $200 / 130$ \\
\hline $\mathrm{Pa}-029$ & - & - & $X$ & $X$ & $\mathrm{X}$ & - & $1 \mathrm{~km}$ \\
\hline $\mathrm{Pa}-030$ & - & - & $\mathrm{X}$ & - & - & - & $30 / 30$ \\
\hline $\mathrm{Pa}-031$ & - & $\mathrm{X}$ & - & $\mathrm{X}$ & $X$ & - & $40 / 20$ \\
\hline
\end{tabular}




\begin{tabular}{|c|c|c|c|c|c|c|c|}
\hline $\mathrm{Pa}-032$ & - & $\mathrm{X}$ & - & - & $\mathrm{X}$ & - & $20 / 20$ \\
\hline $\mathrm{Pa}-033$ & $\mathrm{X}$ & - & - & $?$ & $\mathrm{X}$ & - & $100 / 50$ \\
\hline $\mathrm{Pa}-034$ & - & $\mathrm{X}$ & - & - & $\mathrm{X}$ & - & $80 / 40$ \\
\hline $\mathrm{Pa}-035$ & - & $X$ & - & - & $\mathrm{X}$ & - & $100 / 20$ \\
\hline $\mathrm{Pa}-036$ & - & $X$ & - & - & $\mathrm{X}$ & - & $70 / 30$ \\
\hline $\mathrm{Pa}-037$ & - & $X$ & - & - & - & - & $30 / 30$ \\
\hline $\mathrm{Pa}-038$ & - & $\mathrm{X}$ & - & $\mathrm{X}$ & $\mathrm{X}$ & - & $1 \mathrm{~km} / 80$ \\
\hline $\mathrm{Pa}-039$ & - & $X$ & - & - & $\mathrm{X}$ & - & - \\
\hline $\mathrm{Pa}-040$ & $\mathrm{X}$ & - & - & $\mathrm{X}$ & $\mathrm{X}$ & $?$ & - \\
\hline
\end{tabular}

$\begin{array}{lllllll}\text { Total } & 16 & 21 & 3 & 23 & 38 & 1\end{array}$

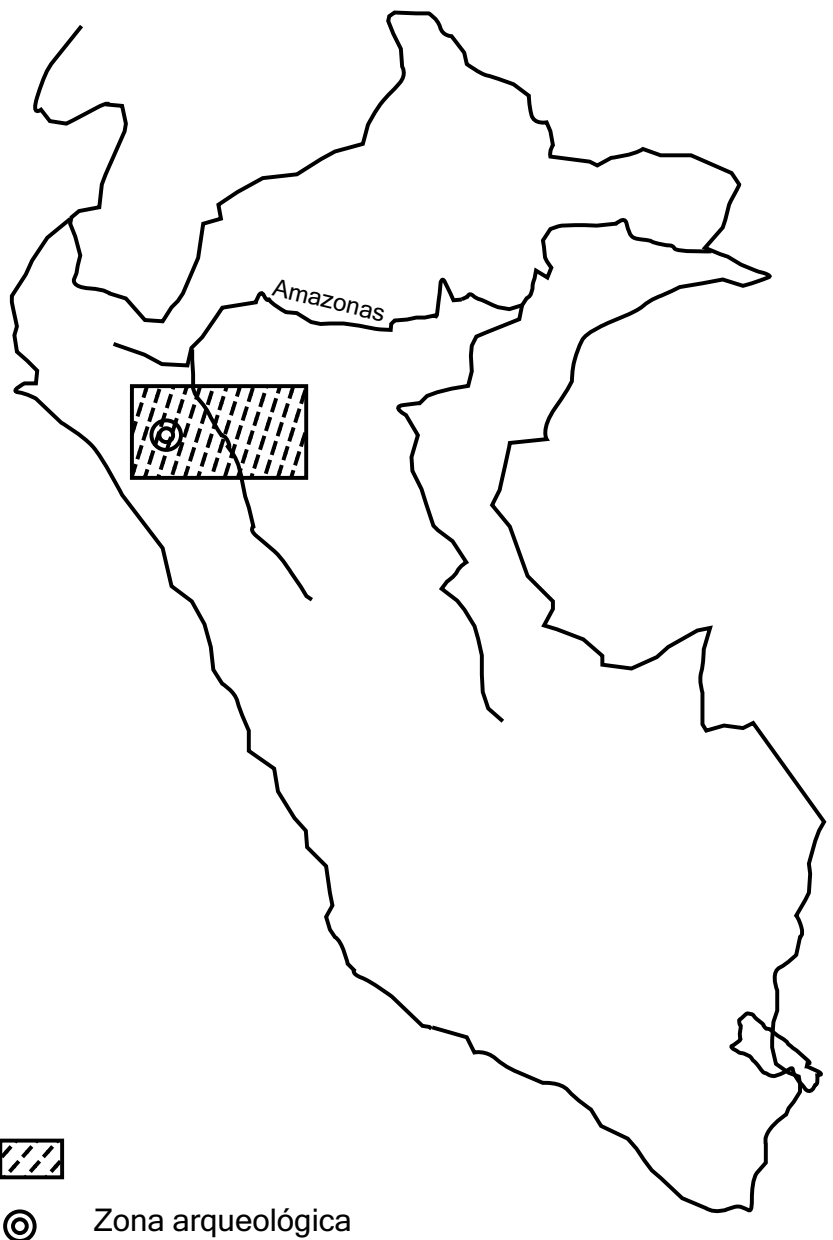

Figura 1. Mapa de ubicación del sitio de Pacopampa 


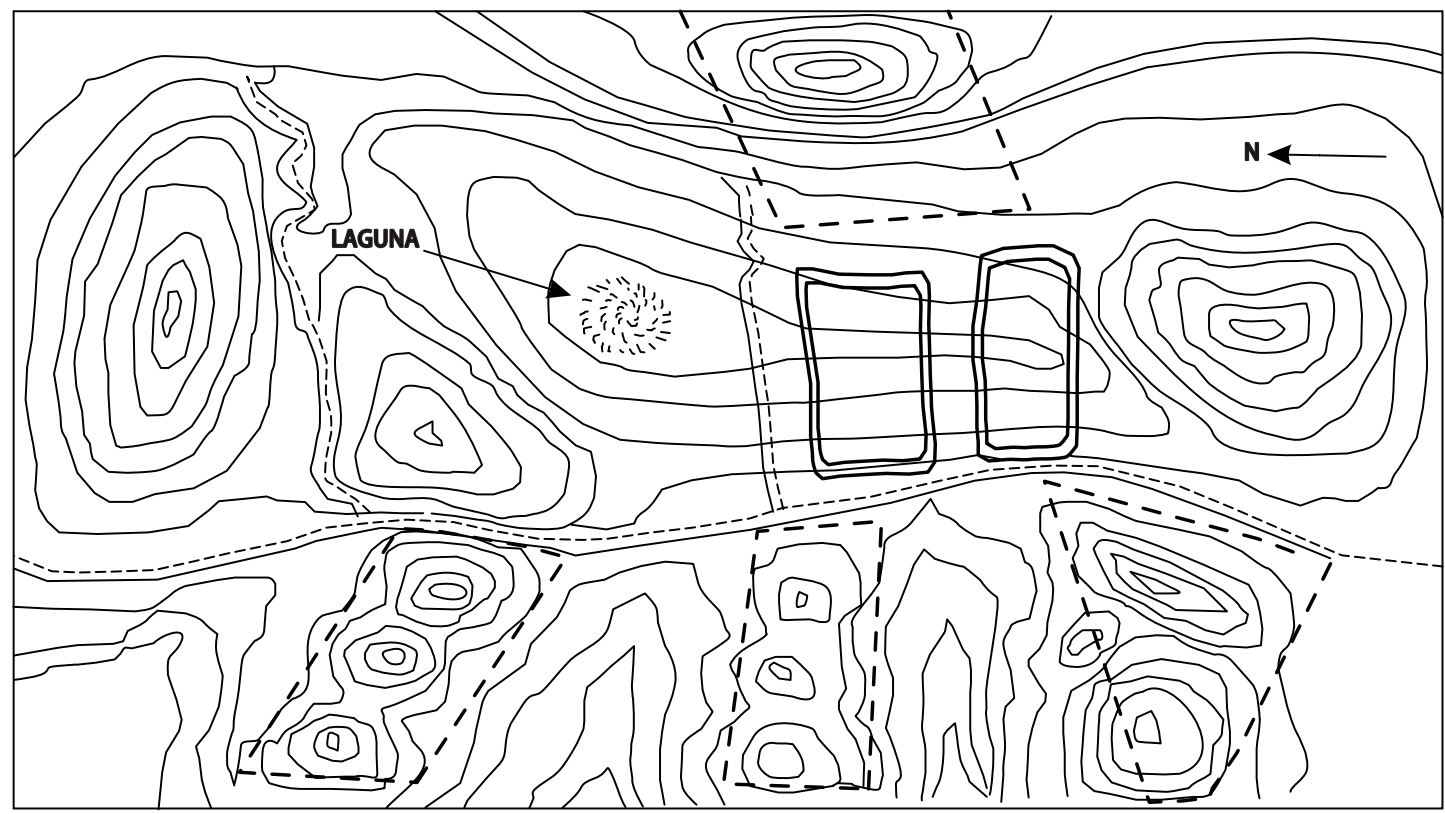

Figura 2. Plano esquemático de la distribución de zonas agrícolas.

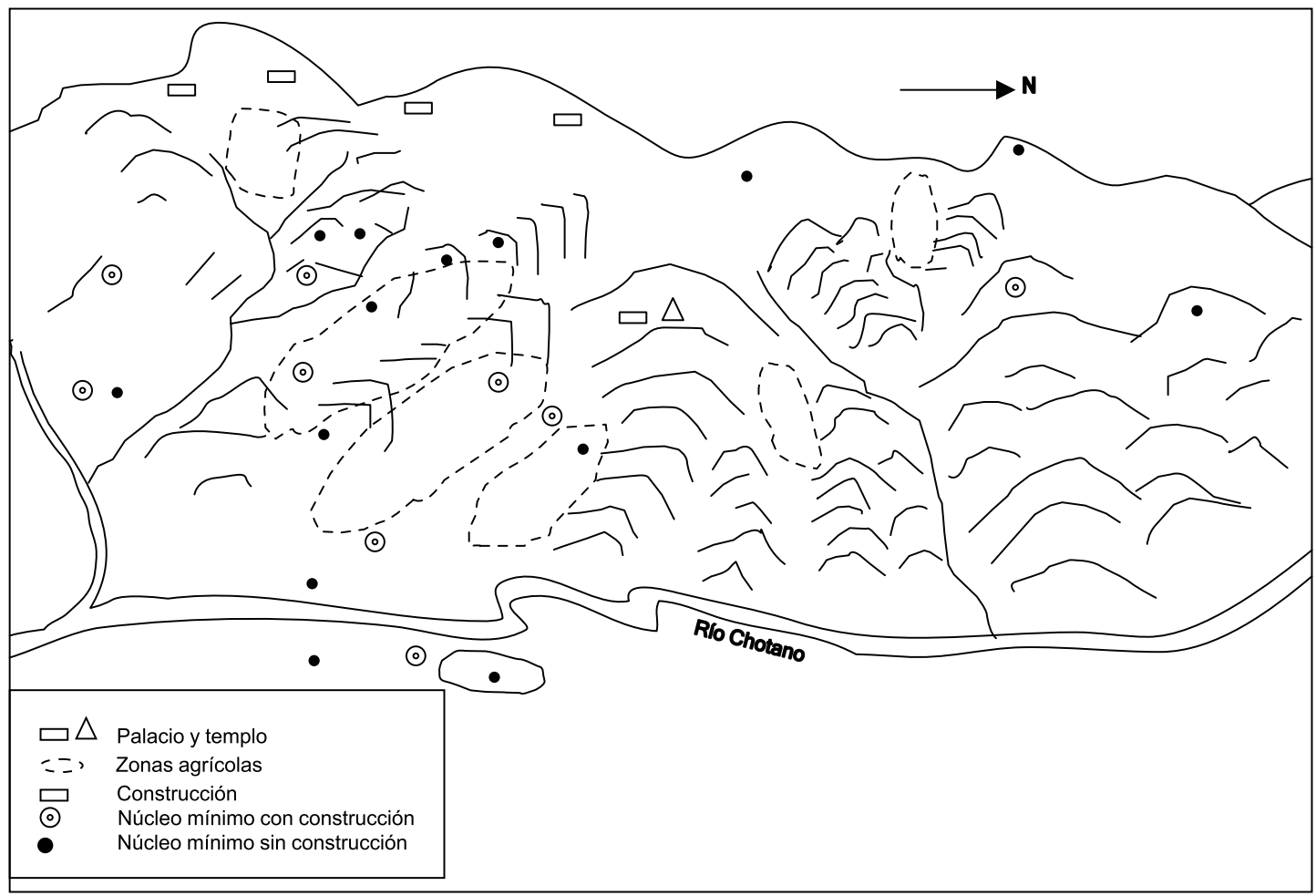

Figura 3. Diagrama de distribución de zonas agrícolas y asentamientos en el entorno del sitio de Pacopampa. 